\title{
THE HEAT EQUATION FOR RIEMANNIAN FOLIATIONS
}

\author{
SEIKI NISHIKAWA, MOHAN RAMACHANDRAN AND \\ PHILIPPE TONDEUR
}

In memory of Bruce L. Reinhart

\begin{abstract}
Let $\mathscr{F}$ be a Riemannian foliation on a closed oriented manifold $M$, with the transversal Laplacian $\Delta_{B}$ acting on the basic forms $\Omega_{B}^{r}(\mathscr{F})$ of degree $r \geq 0$. We construct the fundamental solution $e_{B}^{r}(x, y, t)$ for the basic heat operator $\partial / \partial t+\Delta_{B}$, and prove existence and uniqueness for the solution of the heat equation on $\Omega_{B}^{r}(\mathscr{F})$. As an application we give a new proof for the deRham-Hodge decomposition theorem for $\Delta_{B}$ in $\Omega_{B}^{r}(\mathscr{F})$, generalizing the approach to the classical deRham-Hodge theorem pioneered by Milgram and Rosenbloom.
\end{abstract}

\section{INTRODUCTION}

Let $\mathscr{F}$ be a foliation on a closed oriented manifold $M$ given by an exact sequence of vector bundles

$$
0 \rightarrow L \rightarrow T M \stackrel{\pi}{\rightarrow} Q \rightarrow 0,
$$

where $L$ is the tangent bundle and $Q$ the normal bundle of $\mathscr{F}$. The basic subcomplex $\Omega_{B} \equiv \Omega_{B}(\mathscr{F})$ of the deRham complex $\Omega_{M}$ is given by the forms $\omega$ satisfying $i(X) \omega=0$ and $\theta(X) \omega=0$ for all tangent vector fields $X \in \Gamma L$. The exterior differential $d$ on $\Omega_{M}$ restricts by the Cartan formula $\theta(X)=$ $d i(X)+i(X) d$ to a differential $d_{B}: \Omega_{B}^{\cdot} \rightarrow \Omega_{B}^{+1}$. The basic cohomology $H_{B} \equiv$ $H_{B}(\mathscr{F})=H\left(\Omega_{B}, d_{B}\right)$ plays the role of the deRham cohomology of the leaf space $M / \mathscr{F}$ of the foliation.

Let $\mathscr{F}$ be a Riemannian foliation on $M$. A holonomy invariant transversal metric $g_{Q}$ then gives rise to a basic Laplacian $\Delta_{B}=d_{B} \delta_{B}+\delta_{B} d_{B}: \Omega_{B}^{r} \rightarrow$ $\Omega_{B}^{r}$ for all $r=0, \ldots, q$ up to the codimension $q$ of the foliation. For the purposes of this paper we will use throughout a bundle-like metric $g_{M}$ with basic mean curvature form $\kappa$, i.e., $\kappa \in \Omega_{B}^{1}$ (see $\S 2$ ). The formal adjoint $\delta_{B}: \Omega_{B}^{r} \rightarrow \Omega_{B}^{r-1}$ and therefore the Laplacian $\Delta_{B}: \Omega_{B}^{r} \rightarrow \Omega_{B}^{r}$ explicitly involve the mean curvature form. The harmonic basic forms $\mathscr{H}_{B}^{r}$ of degree $r$ are defined as the kernel of $\Delta_{B}$ on $\Omega_{B}^{r}$.

Received by the editors August 22, 1988.

1980 Mathematics Subject Classification (1985 Revision). Primary 58A14, 58G11.

Key words and phrases. Heat equation, Riemannian foliation.

Work supported in part by a grant from the National Science Foundation. 
For a given form $\alpha_{0} \in \Omega_{B}^{r}$, consider the initial value problem

$$
\frac{\partial}{\partial t} \alpha(x, t)=-\Delta_{B} \alpha(x, t), \quad \lim _{t \downarrow 0} \alpha(x, t)=\alpha_{0}(x) .
$$

The main results of this paper can then be stated as follows.

Theorem. Let $\mathscr{F}$ be a transversally oriented Riemannian foliation on a closed oriented manifold $\left(M, g_{M}\right)$. Assume $g_{M}$ to be a bundle-like metric with $\kappa \in$ $\Omega_{B}^{1}$. Then the following hold.

(i) There exists a unique solution $\alpha$ of (1.1), given in terms of the fundamental solution $e_{B}^{r}(x, y, t)$ of the basic heat operator $\partial / \partial t+\Delta_{B}$ by

$$
\alpha(x, t)=\int_{M} e_{B}^{r}(x, y, t) \wedge * \alpha_{0}(y) .
$$

(ii) Denote $\alpha(x, t)=\left[P_{B}(t) \alpha_{0}\right](x)$. Then there exists a uniform limit

$$
\lim _{t \uparrow \infty} P_{B}(t) \alpha_{0}=H_{B} \alpha_{0} \in \Omega_{B}^{r},
$$

and $H_{B} \alpha_{0}$ is $\Delta_{B}$-harmonic.

(iii) The form

$$
G_{B} \alpha_{0}=\int_{0}^{\infty}\left(P_{B}(t) \alpha_{0}-H_{B} \alpha_{0}\right) d t
$$

is well defined, and gives an operator $G_{B}: \Omega_{B}^{r} \rightarrow \Omega_{B}^{r}$ satisfying

$$
\alpha_{0}=\Delta_{B} G_{B} \alpha_{0}+H_{B} \alpha_{0} .
$$

The finite-dimensionality of $\mathscr{H}_{B}^{r}=\operatorname{ker} \Delta_{B}$ is a consequence of the proofmethod. The identity in (iii) implies in usual fashion the orthogonal decomposition

$$
\Omega_{B}^{r} \cong \operatorname{im} d_{B} \oplus \operatorname{im} \delta_{B} \oplus \mathscr{H}_{B}^{r}
$$

and the isomorphism $H_{B}^{r} \cong \mathscr{H}_{B}^{r}$.

There are two published proofs [EH, KT2] of the deRham-Hodge decomposition theorem in $\Omega_{B}^{r}$. The proof in [EH] does not involve the mean curvature assumption stated above. But the fundamental estimates necessary in certain applications, as, e.g., in [KRT], seem inaccessible through that method. On the other hand, the proofs in [KT2] and in this paper, using an explicit elliptic extension of $\Delta_{B}$ to the full deRham complex, do provide the necessary control to prove the desired estimates.

For the case of a point foliation, the heat equation approach described above is Milgram and Rosenbloom's approach to the classical deRham-Hodge decomposition theorem [MR]. A stochastic interpretation of this can be seen in Yosida [Y]. The new technical aspect in the present context is that the basic forms do not constitute all sections of a vector bundle, and the usual elliptic theory does not apply directly. The technical device to handle this situation is to extend $\Delta_{B}: \Omega_{B}(\mathscr{F}) \rightarrow \Omega_{B}(\mathscr{F})$ to a genuine elliptic operator $\tilde{\Delta}: \Omega_{M} \rightarrow \Omega_{M}$ on the 
full deRham complex, and to exploit the standard elliptic theory for $\tilde{\Delta}$. An explicit construction of such an extension was given in [KT2]. This is where the assumption of the basic mean curvature is involved (see §2). The authors do not know if the construction of the fundamental solution of the heat equation holds in a more general context. But what is known is that the mean curvature hypothesis holds for the following interesting classes of foliations: Riemannian submersions for appropriate metrics, E. Cartan's isoparametric families of surfaces, and foliations by the orbits of isometric Lie group actions. A typical example of the latter kind is the flow defined by a nonsingular Killing field. The mean curvature form is invariant under the flow, hence constant along the orbits.

In the construction of the fundamental solution $e_{B}^{r}(x, y, t)$ of the basic heat operator $\partial / \partial t+\Delta_{B}$, the necessary convergence properties arise from the existence of the strongly elliptic extension $\tilde{\Delta}$ (see $\S 4$ ). However, since $\tilde{\Delta}$ need not be selfadjoint, this does not directly follow from corresponding properties of the fundamental solution of the heat operator $\partial / \partial t+\tilde{\Delta}$. For the case of basic functions (forms of degree 0) treated in [EK] these difficulties do not arise, since the ordinary Laplacian already preserves basic functions. In this case no assumption on the mean curvature is needed.

Comparing the heat flows $e^{-t \Delta_{B}}$ and $e^{-t \tilde{\Delta}}$, an important consequence of these constructions is the Invariance Theorem 4.11, stating that $e^{-t \tilde{\Delta}}$ acting on the full deRham complex $\Omega_{M}$ leaves the subcomplex $\Omega_{B}$ invariant, and coincides on $\Omega_{B}$ with $e^{-t \Delta_{B}}$. This constitutes a parabolic version of Hadamard's descent method for hyperbolic equations $[\mathrm{H}]$.

We expect that this heat equation approach will prove to be useful for the discussion of the transversal index problem for foliations. Another application is given in [RT], where an almost Lie foliation structure is deformed to a Lie foliation structure by the heat flow method.

We wish to contrast this discussion of the heat equation in transversal geometry with the heat flow along the leaves of $\mathscr{F}$. The latter is defined by the leafwise Laplacian $\Delta_{0}$. A deRham-Hodge decomposition for this leafwise elliptic operator has been established in [AT] for an appropriately defined Sobolev space of forms on $M$.

Acknowledgments. The authors are indebted to I. D. Berg, R. Carroll, F. Kamber, P. Newton, T. Ting, and the referee for helpful comments.

\section{CAlculus in $\Omega_{B}$}

The ingredients for the calculus in $\Omega_{B}$ are as follows [KT1, KT2]. Let $\nu \in \Omega_{B}^{q}$ be the transversal (closed) volume form associated to the transversal holonomy invariant Riemannian metric $g_{Q}$. The characteristic form $\chi_{g}$ of $\mathscr{F}$ is the $p$-form ( $p=$ dimension of leaves) given by $\chi_{\mathscr{F}}=* \nu$, expressed in terms of the star operator associated to $g_{M}$. The Riemannian volume form 
$\mu \in \Omega_{M}^{n}$ is then given by $\mu=\nu \wedge \chi_{\mathscr{F}}$. These forms define the compatible orientations used throughout this paper.

The Weingarten map $W(Z): L \rightarrow L$ for $Z \in \Gamma L^{\perp}$ is given by $W(Z) X=$ $-\pi^{\perp}\left(\nabla_{X}^{M} Z\right)$ for $X \in \Gamma L$, where $\nabla^{M}$ denotes the Riemannian connection of $g_{M}$, and $\pi^{\perp}$ the tangential projection to $L$ with kernel $L^{\perp}$. The mean curvature 1 -form $\kappa$ is then defined by $\kappa(Z)=\operatorname{Tr} W(Z)$ for $Z \in \Gamma L^{\perp}$ and $\kappa(X)=0$ for $X \in \Gamma L$ (the factor $1 / p$ has been suppressed throughout). $\chi_{\mathscr{F}}$ and $\kappa$ are related by Rummler's formula

$$
d \chi_{\mathscr{F}}+\kappa \wedge \chi_{\mathscr{F}} \equiv 0 \bmod \mathscr{F} \text {-trivial forms, }
$$

i..e, modulo forms evaluating to zero on $p$-vectors in $\bigwedge^{p} L$.

To give an alternative formulation of $(2.1)$, consider the filtration [KT1, (2.1)]

$$
F^{r} \Omega^{m}=\left\{\omega \in \Omega^{m} \mid i\left(X_{1}\right) \cdots i\left(X_{m-r+1}\right) \omega=0\right\}
$$

for $X_{1}, \ldots, X_{m-r+1} \in \Gamma L$. This decreasing filtration breaks off above the codimension $q$ of $\mathscr{F}$. The $\mathscr{F}$-trivial $(p+r)$-forms are precisely $F^{r+1} \Omega^{p+r}$. Thus $(2.1)$ is equivalent to

$$
d \chi_{\mathscr{F}}+\kappa \wedge \chi_{\mathscr{F}}=\varphi_{0} \in F^{2} \Omega^{p+1} .
$$

We assume throughout that $\kappa \in \Omega_{B}^{1}$. This implies $d \kappa=0$ [KT2, Appendix].

The star operator $*: \Omega_{B}^{r} \rightarrow \Omega_{B}^{q-r}$ associated to $g_{Q}$ is related to the star operator $*: \Omega_{M}^{r} \rightarrow \Omega_{M}^{n-r}$ associated to $g_{M}$ by the formulas

$$
\begin{gathered}
\bar{*} \alpha=(-1)^{p(q-r)} *\left(\alpha \wedge \chi_{\mathscr{F}}\right), \\
* \alpha=\bar{*} \alpha \wedge \chi_{\mathscr{F}}
\end{gathered}
$$

for $\alpha \in \Omega_{B}^{r}$. The global scalar product $\langle$,$\rangle on \Omega_{M}^{r}$ restricts on $\Omega_{B}^{r}$ to the expression

$$
\left\langle\alpha, \alpha^{\prime}\right\rangle_{B}=\int_{M} \alpha \wedge \bar{*} \alpha^{\prime} \wedge \chi_{\mathscr{F}} .
$$

With respect to this scalar product, the formal adjoint $\delta_{B}: \Omega_{B}^{r} \rightarrow \Omega_{B}^{r-1}$ of $d_{B}=d \mid \Omega_{B}$ is given on $\alpha \in \Omega_{B}^{r}$ by [KT1, (4.14)]

$$
\delta_{B} \alpha=(-1)^{q(r+1)+1} \bar{*}\left(d_{B}-\kappa \wedge\right) \bar{*} \alpha .
$$

This is where the hypothesis of a basic mean curvature $\kappa$ is involved. The basic Laplacian is then $\Delta_{B}=\delta_{B} d_{B}+d_{B} \delta_{B}$.

\section{3. $\Delta_{B}$ AND COMPLETE ORTHOGONAL SYSTEM FOR $L^{2}\left(\Omega_{B}\right)$}

The idea in [KT2] is to study $\Delta_{B}$ by constructing a strongly elliptic extension $\tilde{\Delta}: \Omega_{M}^{r} \rightarrow \Omega_{M}^{r}$. Let $\delta: \Omega_{M}^{r} \rightarrow \Omega_{M}^{r-1}$ be the formal adjoint of $d$, which is given by

$$
\delta \omega=(-1)^{n(r+1)+1} * d * \omega .
$$


The relation between $\delta \alpha$ and $\delta_{B} \alpha$ for $\alpha \in \Omega_{B}^{r}$ is then given by [KT2, Proposition 3.3]

$$
\delta \alpha=\delta_{B} \alpha+* \gamma(\alpha)
$$

where

$$
\gamma(\alpha)=(-1)^{(p+1)(r+1)+q r} \bar{*} \alpha \wedge \varphi_{0} .
$$

Let $\tilde{\gamma}: \Omega_{M}^{r} \rightarrow \Omega_{M}^{n-r+1}$ be the bounded linear operator given by [KT2, (3.11)]

$$
\tilde{\gamma}(\omega)=(-1)^{(q+1)(p+r)+1} *\left(\omega \wedge \chi_{\mathscr{F}}\right) \wedge \varphi_{0},
$$

and $\tilde{\eta}: \Omega_{M}^{r} \rightarrow \Omega_{M}^{r}$ the differential operator of order one or less given by [KT2,

$$
\tilde{\eta}(\omega)=* \tilde{\gamma}(d \omega)+d * \tilde{\gamma}(\omega) .
$$

Using the ordinary Laplacian $\Delta=\Delta_{M}: \Omega_{M}^{r} \rightarrow \Omega_{M}^{r}$, now let

$$
\tilde{\Delta}=\Delta-\tilde{\eta}: \Omega_{M}^{r} \rightarrow \Omega_{M}^{r} .
$$

Then $\tilde{\Delta}$ satisfies by [KT2, (3.6)]

$$
\tilde{\Delta} \mid \Omega_{B}=\Delta_{B} .
$$

The main feature of the extension $\tilde{\Delta}$ of $\Delta_{B}$ is that it differs from the ordinary Laplacian $\Delta$ by lower order terms (the correction term $\tilde{\eta}(\omega)$ is of order one or less). In particular the symbol is unchanged and $\tilde{\Delta}$ is strongly elliptic. We use the existence of $\tilde{\Delta}$ in the proof of the following result.

3.6. Proposition. There exists a complete orthonormal system (COS) for $L^{2}\left(\Omega_{B}^{r}\right)$, consisting of smooth eigenforms of $\Delta_{B}$ in $\Omega_{B}^{r}$.

Proof. First we note the obvious identities

$$
\left\langle\Delta_{B} \alpha, \beta\right\rangle=\left\langle\alpha, \Delta_{B} \beta\right\rangle, \quad\left\langle\Delta_{B} \alpha, \alpha\right\rangle=\left\|d_{B} \alpha\right\|_{L^{2}}^{2}+\left\|\delta_{B} \alpha\right\|_{L^{2}}^{2}
$$

for $\alpha, \beta \in \Omega_{B}^{r}$, which imply that $\Delta_{B}$ defines a symmetric, positive operator

$$
\Delta_{B}: D\left(\Delta_{B}\right)=\Omega_{B} \subset L^{2}\left(\Omega_{B}\right) \rightarrow L^{2}\left(\Omega_{B}\right) .
$$

We consider the symmetric operator $\Delta_{1}=\Delta_{B}+I$,

$$
\Delta_{1}: D\left(\Delta_{1}\right)=\Omega_{B} \subset L^{2}\left(\Omega_{B}\right) \rightarrow L^{2}\left(\Omega_{B}\right),
$$

which is semibounded from below with lower bound 1, i.e.,

$$
\left\langle\Delta_{1} \alpha, \alpha\right\rangle \geq\|\alpha\|_{L^{2}}^{2} \text { for } \alpha \in \Omega_{B} .
$$

Let $\Delta_{1}^{F}$ denote the Friedrichs extension of $\Delta_{1}$ [CO, p.11]

$$
\Delta_{1}^{F}: D\left(\Delta_{1}^{F}\right) \subset L^{2}\left(\Omega_{B}\right) \rightarrow L^{2}\left(\Omega_{B}\right),
$$

which by definition is the adjoint $\Delta_{1}^{*}$ of $\Delta_{1}$ restricted to the domain $D\left(\Delta_{1}^{F}\right)=$ $D\left(\Delta_{1}^{*}\right) \cap H_{1}$. Here $H_{1}$ denotes the Hilbert space completion of $\Omega_{B}$ with respect to the scalar product

$$
\langle\alpha, \beta\rangle_{1}=\left\langle\Delta_{1} \alpha, \beta\right\rangle \text { for } \alpha, \beta \in \Omega_{B} .
$$


Note that since

$$
\|\alpha\|_{1}^{2}=\left\langle\Delta_{1} \alpha, \alpha\right\rangle=\left\|d_{B} \alpha\right\|_{L^{2}}^{2}+\left\|\delta_{B} \alpha\right\|_{L^{2}}^{2}+\|\alpha\|_{L^{2}}^{2},
$$

the new norm \|\|$_{1}$ is equivalent to the Sobolev $H_{1}$-norm on $\Omega_{B}$; hence $H_{1} \cong H_{1}\left(\Omega_{B}\right)$.

It follows from its construction that the Friedrichs extension $\Delta_{1}^{F}$ is still symmetric and surjective, and hence selfadjoint. Furthermore, since $\Delta_{1}^{F}$ has the same lower bound 1 as $\Delta_{1}, \Delta_{1}^{F}$ is also injective. Hence we have the selfadjoint inverse

$$
G_{1}^{F}=\left(\Delta_{1}^{F}\right)^{-1}: R\left(\Delta_{1}^{F}\right)=L^{2}\left(\Omega_{B}\right) \rightarrow D\left(\Delta_{1}^{F}\right) \subset L^{2}\left(\Omega_{B}\right),
$$

which is a bounded map into $D\left(\Delta_{1}^{F}\right)$. Since by Rellich's lemma the inclusion $H_{1} \cong H_{1}\left(\Omega_{B}\right) \hookrightarrow L^{2}\left(\Omega_{B}\right)$ is compact, the composition

$$
G_{1}^{F}: L^{2}\left(\Omega_{B}\right) \rightarrow L^{2}\left(\Omega_{B}\right)
$$

is a compact selfadjoint operator. Thus $G_{1}^{F}$ has eigenvalues $\left|\mu_{1}\right| \geq\left|\mu_{2}\right| \geq \cdots \downarrow$ 0 with corresponding eigenforms $\left\{\psi_{i}\right\}$ constituting a $\operatorname{COS}$ for $L^{2}\left(\Omega_{B}\right)$ [GL, p. 43].

We prove now that the $\psi_{i}$ are smooth eigenforms of $\Delta_{B}$. First note that $G_{1}^{F} \psi_{i}=\mu_{i} \psi_{i}$ implies that $\psi_{i} \in D\left(\Delta_{1}^{F}\right) \subset H_{1}\left(\Omega_{B}\right)$ and $\Delta_{1}^{F} \psi_{i}=\mu_{i}^{-1} \psi_{i}$. Now consider the elliptic operator $\tilde{\Delta}_{1}=\tilde{\Delta}+I$, and let $\tilde{\Delta}_{1}^{*}$ denote its formal adjoint. Then we observe by the definition of distribution derivatives and (3.5) that for any $\alpha \in \Omega_{B}$

$$
\left\langle\tilde{\Delta}_{1} \psi_{i}, \alpha\right\rangle=\left\langle\psi_{i}, \tilde{\Delta}_{1}^{*} \alpha\right\rangle=\left\langle\psi_{i}, \Delta_{1} \alpha\right\rangle=\left\langle\Delta_{1}^{F} \psi_{i}, \alpha\right\rangle=\left\langle\mu_{i}^{-1} \psi_{i}, \alpha\right\rangle ;
$$

that is, $\left\langle\left(\tilde{\Delta}_{1}-\mu_{i}^{-1} I\right) \psi_{i}, \alpha\right\rangle=0$ for any $\alpha \in \Omega_{B}$. Note that $\left(\tilde{\Delta}_{1}-\mu_{i}^{-1} I\right) \psi_{i} \in$ $H_{-1}\left(\Omega_{B}\right)$, the completion of $\Omega_{B}$ with respect to the Sobolev $H_{-1}$-norm \|\|$_{-1}$ on $\Omega_{B}$ defined by

$$
\|\alpha\|_{-1}=\sup \left\{|\langle\alpha, \beta\rangle|\|\beta\|_{1} \leq 1, \beta \in \Omega_{B}\right\} .
$$

Since the pairing $\langle\rangle:, H_{-1}\left(\Omega_{B}\right) \times H_{1}\left(\Omega_{B}\right) \rightarrow \mathbf{R}$ is continuous and nondegenerate, it then follows that $\psi_{i}$ satisfies the elliptic equation

$$
\left(\tilde{\Delta}_{1}-\mu_{i}^{-1} I\right) \psi_{i}=0 .
$$

This implies the regularity of $\psi_{i}$ [GL, p. 23]; that is, $\psi_{i} \in \Omega_{M}$ and hence $\psi_{i} \in H_{1}\left(\Omega_{B}\right) \cap \Omega_{M}$. Since by [KT2, Corollary 4.14] we have $H_{1}\left(\Omega_{B}\right) \cap \Omega_{M}=\Omega_{B}$, this implies $\psi_{i} \in \Omega_{B}$. Now $\tilde{\Delta}_{1} \psi_{i}=\mu_{i}^{-1} \psi_{i}$ implies by (3.5) that $\Delta_{B} \psi_{i}=\lambda_{i} \psi_{i}$ with $\lambda_{i}=\mu_{i}^{-1}-1$.

Note that since $\Delta_{B}$ is a positive operator, we have $\lambda_{i} \geq 0$. From $\left|\mu_{1}\right| \geq$ $\left|\mu_{2}\right| \geq \cdots \downarrow 0$ it follows that $0 \leq \lambda_{1} \leq \lambda_{2} \leq \cdots \uparrow \infty$ (except of course for the case of a finite-dimensional $\Omega_{B}^{r}$, an exception we will not point out repeatedly). Furthermore, the multiplicities of the eigenvalues $\lambda_{i}$ cannot increase too rapidly by the following fact. 
3.7. Lemma. Let $0 \leq \lambda_{1} \leq \lambda_{2} \leq \cdots \uparrow \infty$ be the eigenvalues of $\Delta_{B}$ in $\Omega_{B}^{r}$. Then there exists a constant $C>0$ and an exponent $\delta>0$ such that $\lambda_{j} \geq C \cdot j^{\delta}$, provided $j \geq j_{0}$ is large.

This is proved by the same argument as in [GL, p. 44].

\section{Heat equation}

For a given initial $r$-form $\alpha_{0} \in \Omega_{B}^{r}$, we consider the initial value problem for the heat equation on $\Omega_{B}^{r}$ :

$$
\frac{\partial}{\partial t} \alpha(x, t)=-\Delta_{B} \alpha(x, t), \quad \lim _{t \downarrow 0} \alpha(x, t)=\alpha_{0}(x) .
$$

We are going to prove that (4.1) has a unique solution $\alpha(x, t) \in \Omega_{B}^{r}, t>0$, which is given in terms of the fundamental solution $e_{B}^{r}(x, y, t)$ of the basic heat operator $L_{B}=\partial / \partial t+\Delta_{B}$, to be constructed below, by

$$
\alpha(x, t)=\int_{M} e_{B}^{r}(x, y, t) \wedge * \alpha_{0}(y) .
$$

For $(x, y, t) \in M \times M \times(0, \infty)$ we define

$$
e_{B}^{r}(x, y, t)=\sum_{i=1}^{\infty} e^{-\lambda_{i} t} \psi_{i}(x) \otimes \psi_{i}(y) \in \bigwedge^{r} T_{x}^{*} M \otimes \bigwedge^{r} T_{y}^{*} M,
$$

where $\left\{\psi_{i}\right\}_{i=1}^{\infty}$ is a COS for $L^{2}\left(\Omega_{B}^{r}\right)$ consisting of smooth eigenforms $\psi_{i}$ of $\Delta_{B}$ corresponding to the eigenvalues $\lambda_{i}$ (Proposition 3.6).

4.4. Proposition. $e_{B}^{r}(x, y, t)$ is a well-defined smooth double form of bidegree $(r, r)$ on $M \times M$, depending smoothly on the parameter $t>0$.

Proof. Let $k$ and $s$ be positive integers such that $2 s>k+n / 2$. Then, by the a priori coercive estimate for $\tilde{\Delta}$ combined with the Sobolev embedding lemma, we get the fundamental estimate

$$
\left\|\psi_{i}\right\|_{C^{k}} \leq C\left(\left\|\tilde{\Delta}^{s} \psi_{i}\right\|_{L^{2}}+\left\|\psi_{i}\right\|_{L^{2}} \leq C\left(1+\lambda_{i}^{s}\right) .\right.
$$

Note also that for $t>0$ and $\lambda>0$ we have the estimate

$$
e^{-t \lambda} \lambda^{s} \leq t^{-s} C(s) e^{-t \lambda / 2}
$$

By applying these two inequalities to the RHS in (4.3), we get the following estimate:

$$
\left\|e_{B}^{r}(x, y, t)\right\|_{C^{k}} \leq t^{-s(k)} C(k) \sum_{i=1}^{\infty} e^{-t \lambda_{i} / 2}
$$

But by Lemma 3.7, for large $j>j_{0}$, we have also the estimate $\lambda_{j}>C \cdot j^{\delta}$ for some $C>0$ and $\delta>0$. This implies that the series on the RHS of (4.5) can be bounded by (some constant multiple of) the series $\sum_{j=1}^{\infty} e^{-t \cdot j^{\delta} / 2}$, which converges absolutely for each $t>0$. Since $k$ is arbitrary, this shows that $e_{B}^{r}(x, y, t)$ is a well-defined smooth $(r, r)$-form on $M \times M \times(0, \infty)$. 
Note that the integrand in (4.2) is a double form of bidegree $(r, n)$, which integrates for fixed $x$ and $t$ to an element in $\wedge^{r} T_{x}^{*} M$. It is now verified without difficulty that $\alpha(x, t)$ in (4.2) gives rise to a solution of the initial value problem (4.1).

For $\alpha \in \Omega_{B}^{r}$ and $t>0$ we now define

$$
\left[P_{B}(t) \alpha\right](x)=\int_{M} e_{B}^{r}(x, y, t) \wedge * \alpha(y)=\sum_{i=1}^{\infty} e^{-\lambda_{i} t}\left\langle\alpha, \psi_{i}\right\rangle_{B} \psi_{i}(x) .
$$

Note that $P_{B}(t): \Omega_{B}^{r} \rightarrow \Omega_{B}^{r}$ for all $t>0$. Moreover $P_{B}(t)$ is a symmetric operator for all $t>0$ :

$$
\left\langle P_{B}(t) \alpha, \beta\right\rangle_{B}=\left\langle\alpha, P_{B}(t) \beta\right\rangle_{B} \text { for } \alpha, \beta \in \Omega_{B}^{r} .
$$

In fact, let $f(s, t)=\left\langle P_{B}(t) \alpha, P_{B}(s) \beta\right\rangle_{B}$. Then

$$
\begin{aligned}
\frac{\partial}{\partial t} f(t, s) & =\left\langle\frac{\partial}{\partial t} P_{B}(t) \alpha, P_{B}(s) \beta\right\rangle_{B}=\left\langle-\Delta_{B} P_{B}(t) \alpha, P_{B}(s) \beta\right\rangle_{B} \\
& =\left\langle P_{B}(t) \alpha,-\Delta_{B} P_{B}(s) \beta\right\rangle_{B}=\left\langle P_{B}(t) \alpha, \frac{\partial}{\partial s} P_{B}(s) \beta\right\rangle_{B}=\frac{\partial}{\partial s} f(t, s) .
\end{aligned}
$$

It follows that $f(t, s)$ is a function of $t+s$. For $s=0$ this yields the desired result in the form $f(t, 0)=f(0, t)$.

For a given $\omega_{0} \in \Omega_{M}^{r}$, we can also consider the initial value problem for the heat equation on $\Omega_{M}^{r}$ :

$$
\frac{\partial}{\partial t} \omega(x, t)=-\tilde{\Delta} \omega(x, t), \quad \lim _{t \downarrow 0} \omega(x, t)=\omega_{0}(x) .
$$

It is well known, see, e.g., [GR], that the fundamental solution $e_{M}^{r}(x, y, t)$ for the heat operator $\partial / \partial t+\tilde{\Delta}$ exists, and that the unique solution of $(4.8)$ is given by

$$
\omega(x, t)=\int_{M} e_{M}^{r}(x, y, t) \wedge * \omega_{0}(y) .
$$

For $\omega \in \Omega_{M}^{r}$ and $t>0$ let

$$
[P(t) \omega](x)=\int_{M} e_{M}^{r}(x, y, t) \wedge * \omega(y) .
$$

This defines $P(t): \Omega_{M}^{r} \rightarrow \Omega_{M}^{r}$. The following result may be considered a parabolic analog of Hadamard's descent method for hyperbolic equations $[\mathrm{H}]$.

4.11. Invariance Theorem. (i) If $\alpha \in \Omega_{B}^{r}$, then $P(t) \alpha \in \Omega_{B}^{r}$ for all $t>0$.

(ii) $P(t) \mid \Omega_{B}=P_{B}(t)$ for all $t>0$.

Proof. For a given $\alpha \in \Omega_{B}$, we have a solution $P_{B}(t) \alpha$ of (4.1), and a solution $P(t) \alpha$ of (4.8). Since $P_{B}(t) \alpha \in \Omega_{B}$ and $\tilde{\Delta} \mid \Omega_{B}=\Delta_{B}$, it follows that $P_{B}(t) \alpha$ is also a solution of (4.8). By the uniqueness of the solution to (4.8), it follows that $P(t) \alpha=P_{B}(t) \alpha$. This proves (i) and (ii). 
4.12. Corollary. The solution to the initial value problem (4.1) is unique. In particular, $P_{B}(t)$ has the semigroup property

$$
P_{B}\left(t_{1}\right) \circ P_{B}\left(t_{2}\right)=P_{B}\left(t_{1}+t_{2}\right) \text { for } t_{1}, t_{2}>0 \text {. }
$$

It is now easy to prove the following supplementary fact:

$$
\alpha \in \Omega_{B}^{r}, \quad d_{B} \alpha=0 \Rightarrow d_{B}\left(P_{B}(t) \alpha\right)=0 \text { for all } t \geq 0 .
$$

Proof. By assumption $d_{B} P_{B}(0) \alpha=d_{B} \alpha=0$. We show that $d_{B} P_{B}(t) \alpha$ is a solution of the heat equation (4.1). Namely

$$
\frac{d}{d t} d_{B} P_{B}(t) \alpha=d_{B}\left(\frac{d}{d t} P_{B}(t) \alpha\right)=d_{B}\left(-\Delta_{B} P_{B}(t) \alpha\right)=-\Delta_{B} d_{B} P_{B}(t) \alpha
$$

By the uniqueness of the solution of the initial value problem (4.1), it follows that $d_{B} P_{B}(t) \alpha=0$ for all $t \geq 0$.

\section{LONG TIME BEHAVIOR OF SOLUTIONS}

We now examine $P_{B}(t) \alpha$ for $\alpha \in \Omega_{B}$ in its dependence on $t$.

5.1. Lemma. Let $\alpha \in \Omega_{B}^{r}$. Then $\left\|P_{B}(t) \alpha\right\|$ is a nonincreasing function of $t$ for $t>0$.

Proof. We have

$$
\begin{aligned}
\frac{d}{d t}\left\|P_{B}(t) \alpha\right\|^{2} & =2\left\langle\frac{d}{d t} P_{B}(t) \alpha, P_{B}(t) \alpha\right\rangle=-2\left\langle\Delta_{B} P_{B}(t) \alpha, P_{B}(t) \alpha\right\rangle \\
& =-2\left[\left\|d_{B} P_{B}(t) \alpha\right\|^{2}+\left\|\delta_{B} P_{B}(t) \alpha\right\|^{2}\right] \leq 0 .
\end{aligned}
$$

Next we turn to the discussion of the behavior of $P_{B}(t) \alpha$ for $t \rightarrow \infty$.

5.2. Theorem. Let $\alpha \in \Omega_{B}^{r}$.

(i) $P_{B}(t) \alpha$ converges uniformly for $t \uparrow \infty$.

(ii) $H_{B} \alpha=\lim _{t \uparrow \infty} P_{B}(t) \alpha \in \Omega_{B}^{r}$ is $\Delta_{B}$-harmonic.

Proof. (i) By Lemma 5.1, there exists $\lim _{t \uparrow \infty}\|P(t) \alpha\|^{2}=a \geq 0$. But by Corollary 4.12 and $(4.7)$

$$
\begin{aligned}
& \left\|P_{B}(t+2 h) \alpha-P_{B}(t) \alpha\right\|^{2} \\
& \quad=\left\|P_{B}(t+2 h) \alpha\right\|^{2}+\left\|P_{B}(t) \alpha\right\|^{2}-2\left\langle P_{B}(t+2 h) \alpha, P_{b}(t) \alpha\right\rangle \\
& \quad=\left\|P_{B}(t+2 h) \alpha\right\|^{2}+\left\|P_{B}(t) \alpha\right\|^{2}-2\left\|P_{B}(t+h) \alpha\right\|^{2},
\end{aligned}
$$

which approaches 0 for $t \uparrow \infty$. Thus $\left\{P_{B}(t) \alpha\right\}_{t \geq 0}$ has the Cauchy property, hence converges in $L^{2}\left(\Omega_{B}^{r}\right)$ for $t \uparrow \infty$.

To prove uniform convergence, we fix $h>0$. Then we have for all $t \in(0, \infty)$ and $x \in M$

$$
\begin{aligned}
\mid P_{B}(t & +2 h) \alpha-P_{B}(t+h) \alpha|(x)=| P_{B}(h)\left(P_{B}(t+h) \alpha-P_{B}(t) \alpha\right) \mid(x) \\
& =\left|\int_{M} e_{B}^{r}(x, y, h) \wedge *\left(P_{B}(t+h) \alpha-P_{B}(t) \alpha\right)(y)\right| \\
& \leq \sup _{x \in M}\left\|e_{B}^{r}(x, \cdot h)\right\| \cdot\left\|P_{B}(t+h) \alpha-P_{B}(t) \alpha\right\|,
\end{aligned}
$$


which goes to 0 for $t \uparrow \infty$ by the preceding observation. It follows that $P_{B}(t) \alpha$ converges uniformly on $M$ for $t \uparrow \infty$. We define $H_{B} \alpha$ as this uniform limit.

(ii) To prove that $H_{B} \alpha$ is $\Delta_{b}$-harmonic, we observe first that for fixed $t>0$

$$
\begin{aligned}
\left|P_{B}(t+h) \alpha-P_{B}(t) H_{B} \alpha\right|(x) & =\left|P_{B}(t)\left(P_{B}(h) \alpha-H_{B} \alpha\right)\right|(x) \\
& \leq \sup _{x \in M}\left\|e_{B}^{r}(x, \cdot, t)\right\| \cdot\left\|P_{B}(h) \alpha-H_{B} \alpha\right\|,
\end{aligned}
$$

which implies for $h \uparrow \infty$.

$$
0=\lim _{h \uparrow \infty}\left(P_{B}(t+h) \alpha-P_{B}(t) H_{B} \alpha\right)=H_{B} \alpha-P_{B}(t) H_{B} \alpha .
$$

Hence we have the invariance property

$$
P_{B}(t) H_{B} \alpha=H_{B} \alpha .
$$

It now follows from (5.3) that

$$
\Delta_{B} H_{B} \alpha=\Delta_{B} P_{B}(t) H_{B} \alpha=-\frac{\partial}{\partial t} P_{B}(t) H_{B} \alpha=-\frac{\partial}{\partial t} H_{B} \alpha=0
$$

and $H_{B} \alpha$ is indeed $\Delta_{B}$-harmonic.

Note that since $\tilde{\Delta} \mid \Omega_{B}=\Delta_{B}$, the $\Delta_{B}$-harmonic forms $\mathscr{H}_{B}$ form a subspace of the finite-dimensional space $\mathscr{H}$ of $\tilde{\Delta}$-harmonic forms. Thus $\mathscr{H}_{B}$ is itself finite-dimensional.

For $\alpha \in \Omega_{B}^{r}$ define now

$$
G_{B} \alpha(x)=\int_{0}^{\infty}\left(P_{B}(t) \alpha-H_{B} \alpha\right)(x) d t .
$$

5.5. Lemma. $G_{B} \alpha$ is well defined, and $G_{B} \alpha \in \Omega_{B}^{r}$.

Proof. Let $k=\operatorname{dim} \mathscr{H}_{B}^{r}$. Then in (4.3) we have $\lambda_{1}=\cdots=\lambda_{k}=0$ and $\psi_{1}, \ldots, \psi_{k} \in \mathscr{H}_{B}^{r}$. It follows from (4.6) that

$$
H_{B} \alpha=\lim _{t \uparrow \infty} P_{B}(t) \alpha=\sum_{i=1}^{k}\left\langle\psi_{i}, \alpha\right\rangle_{B} \psi_{i} \text { in } \Omega_{B}^{r},
$$

since the contribution of the positive eigenvalues $\lambda_{k+1} \leq \lambda_{k+2} \leq \cdots$ disappears for $t \uparrow \infty$. Hence we have

$$
P_{B}(t) \alpha-H_{b} \alpha=\sum_{i=k+1}^{\infty} e^{-\lambda_{i} t}\left\langle\psi_{i}, \alpha\right\rangle_{B} \psi_{i} \text { in } \Omega_{B}^{r} .
$$


For fixed $h>0$, and each $x \in M, t \in(0, \infty)$ we then have

$$
\begin{aligned}
\mid P_{B}(t & +h) \alpha-H_{B} \alpha|(x)=| P_{B}(h)\left(P_{B}(t) \alpha-H_{B} \alpha\right) \mid(x) \\
& =\left|\int_{M} e_{B}^{r}(x, y, h) \wedge *\left(P_{B}(t) \alpha-H_{B} \alpha\right)(y)\right| \\
& \leq\left(\sup _{x}\left\|e_{B}^{r}(x, \cdot, h)\right\|\right) \cdot\left\|\left(P_{B}(t) \alpha-H_{B} \alpha\right)\right\| \\
& =c\left\|\sum_{i=k+1}^{\infty} e^{-\lambda_{i} t}\left\langle\psi_{i}, \alpha\right\rangle_{B} \psi_{i}\right\| \\
& \leq c e^{-\lambda_{k+1} t}\left\|\sum_{i}\left\langle\psi_{i}, \alpha\right\rangle_{B} \psi_{i}\right\| \leq c\|\alpha\| e^{-\lambda_{k+1} t} .
\end{aligned}
$$

It follows that

$$
\begin{aligned}
\left|\int_{0}^{t+h}\left(P_{B}(t+h) \alpha-H_{B} \alpha\right)(x) d t\right| & \leq \int_{0}^{t+h}\left|P_{B}(t+h) \alpha-H_{B} \alpha\right|(x) d t \\
& \leq c\|\alpha\| \cdot \int_{0}^{t+h} e^{-\lambda_{k+1} t} d t .
\end{aligned}
$$

Since the last term converges as $t \uparrow \infty$, it follows that the RHS in (5.4) exists as a limit for $t \uparrow \infty$ of $\int_{0}^{t}$, uniformly in $x$. Since the integrand is a basic $r$-form for each $t$, it follows that $G_{B} \alpha \in \Omega_{B}^{r}$.

Finally we calculate, using the harmonicity of $H_{B} \alpha$,

$$
\begin{aligned}
\Delta_{B} G_{B} \alpha & =\Delta_{B} \int_{0}^{\infty}\left(P_{B}(t) \alpha-H_{B} \alpha\right) d t=\int_{0}^{\infty} \Delta_{B}\left(P_{B}(t) \alpha-H_{B} \alpha\right) d t \\
& =\int_{0}^{\infty}\left(\Delta_{B} P_{B}(t) \alpha\right) d t=\int_{0}^{\infty}\left(-\frac{d}{d t} P_{B}(t) \alpha\right) d t \\
& =\lim _{t \downarrow 0} P_{B}(t) \alpha-\lim _{t \uparrow \infty} P_{B}(t) \alpha=\alpha-H_{B} \alpha .
\end{aligned}
$$

The identity

$$
\alpha=\Delta_{B} G_{B} \alpha+H_{B} \alpha
$$

completes now the proof of the theorem stated in the Introduction.

\section{REFERENCES}

[AT] J. A. Alvares-Lópes and $\mathrm{Ph}$. Tondeur, Hodge decomposition along the leaves of a riemannian foliation (to appear).

[CH] I. Chavel, Eigenvalues in Riemannian geometry, Academic Press, 1984.

[CO] H. O. Cordes, Spectral theory of linear differential operators and comparison algebras, London Math. Soc. Lecture Notes Ser., no. 76, Cambridge Univ. Press, 1987.

[EH] A. El Kacimi and G. Hector, Décomposition de Hodge basique pour un feuilletage riemannien, Ann. Inst. Fourier (Grenoble) 36 (1986), 207-227.

[EK] A. El Kacimi, Equation de la chaleur sur les espaces singuliers, C. R. Acad. Sci. Paris Sér. I Math. 303 (1986), 243-246. 
[GL] P. Gilkey, Invariance theory, the heat equation, and the Atiyah-Singer index theorem, Math. Lecture Ser. 11, Publish or Perish, 1984.

[GR] P. Greiner, An asymptotic expansion for the heat equation, Arch. Rational Mech. Anal. 41 (1971), 163-218.

[H] J. Hadamard, Le problème de Cauchy et les équations aux derivées partielles linéaires hypérboliques, Hermann, Paris, 1932.

[KRT] F. W. Kamber, E. A. Ruh, and Ph. Tondeur, Comparing Riemannian foliations with transversally symmetric foliations, J. Differential Geom. 27 (1988), 461-475.

[KT1] F. W. Kamber and Ph. Tondeur, Foliations and metrics, Proc. 1981-82 Year in Differential Geom. (Univ. of Maryland), Progr. Math., vol. 32, Birkhäuser, 1983, pp. 103-152.

[KT2] _ DeRham-Hodge theory for Riemannian foliations, Math. Ann. 277 (1987), 415-431.

[MR] A. N. Milgram and P. C. Rosenbloom, Harmonic forms and heat conduction. I: Closed Riemannian manifolds, Proc. Nat. Acad. Sci. U.S.A. 37 (1951), 180-184.

[RT] E. A. Ruh and Ph. Tondeur, Almost Lie foliations and the heat equation method, Proc. VI Internat. Colloq. on Differential Geometry, Santiago de Compostela (Spain, 1988), Cursos y Congresos 10, Univ. Santiago de Compostela, 1989, pp. 239-246.

[T] Ph. Tondeur, Foliations on Riemannian manifolds, Springer Universitext, 1988.

[Y] K. Yosida, An ergodic theorem associated with harmonic integrals, Proc. Japan Acad. Ser. A Math. Sci. 27 (1951), 540-543.

Department of Mathematics, Faculty of Science, Kyushu University, Fukuoka, 812 JAPAN (Current address of S. Nishikawa)

Department of Mathematics, The Ohio State University, 231 West 18th Avenue, Columbus, Ohio 43210

Department of Mathematics, University of Illinois, 1409 W. Green Street, Urbana, ILLINOIS 61801 (Current address of M. Ramachandran and Philippe Tondeur) 